\section{SAVE A FORTUNE IN LAB FEES}

Buying a CEREC System from Ceramic Systems, the UK CEREC specialists, will enable you to cut down your laboratory bills by up to $90 \%$.

CEREC enables dentists to create high quality and durable chairside all-ceramic restorations in the most cost-effective and efficient way. Quick, efficient and easy to use, CEREC is a computeraided method for creating precision fitting allceramic restorations; saving virtually all your laboratory costs it enables dentists to design and create all-ceramic inlays, onlays, partial crowns, veneers and crowns for the anterior, premolar and molar regions in-house in one visit. Eliminating the need for messy and expensive impressions, CEREC uses a digital impression taking technique to capture the data used to design the restoration which is then milled in the milling unit. The milling unit can be situated anywhere that is convenient within the practice, even as a fascinating eye-catcher in the waiting room.

Combined with adhesive bonding techniques, CEREC creates biocompatible, non-metallic, natural-looking restorations from durable high-quality ceramic materials in a single treatment session - without the need for provisional restorations.

For further information contact Ian Pinner on 01932 582905, email i.pinner@ceramicsystems. co.uk or visit www.ceramicsystems.co.uk.

\section{SIGNIFICANTLY SUPERIOR FLEXURAL STRENGTH}

An article in Clinical Oral Investigations has concluded that ChemFil Rock offers high flexural strength and no visible surface defects.

The authors evaluated the mechanical behaviour and effect of resin coating and storage conditions of ChemFil Rock compared with three conventional glass ionomers. ChemFil Rock fared very well, exhibiting significantly superior flexural strength without resin coating; significantly superior flexural strength with resin coating; and higher flexural strength when resin coating was added.

The article, Evaluation of a conventional glass ionomer cement with new zinc formulation: effect of coating, aging and storage agents (Clin Oral Investig 2012; Epub ahead of print) concluded that 'ChemFil Rock might represent a promising approach regarding longevity of GI fillings in molar regions, due to the high flexural strength and the absence of visible surface defects like crazing and voids'. The impact of storage agent and duration on the measured properties was low. www.dentsply.co.uk

\title{
AN INCOMPARABLE LEVEL OF PERFORMANCE
}

Bien-Air's MCX micromotor is lifetime-lubricated with no maintenance required. It's the

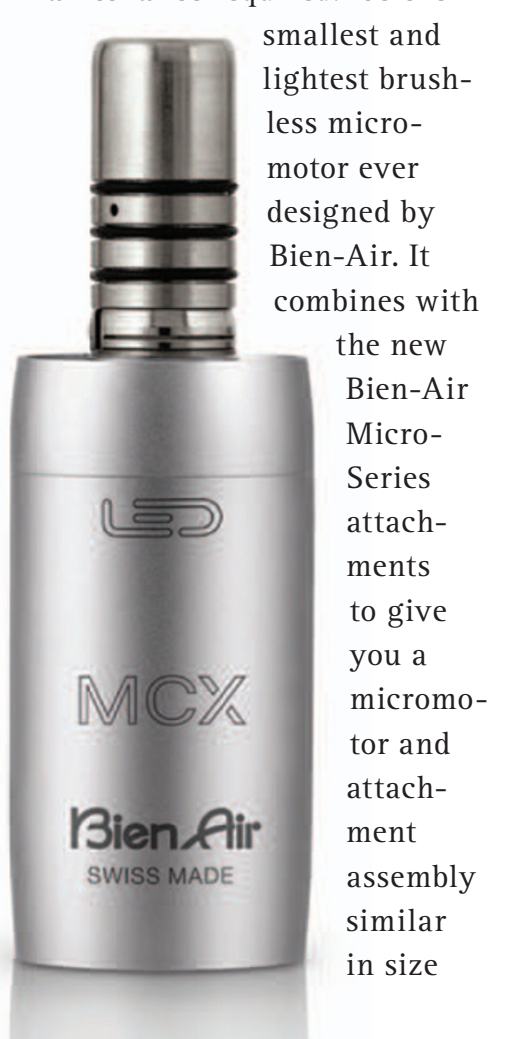

to a turbine, for an incomparable level of performance. With the MCX and Micro-Series you benefit from unprecedented grip and balance, guaranteeing more precise control and less fatigue at the end of the day. MCX and MCX LED: Brushless micromotors, 1,000-40,000 rpm, max. torque $2.5 \mathrm{Ncm}$. Adjustable LED light (MCX LED only) and internal spray. Sterilisable without protection, lifetime-lubricated ball bearings compatible with Type E coupling as per ISO 3964, and Micro-Series attachments.

Competitively priced, the Swiss made MCX from Bien-Air guarantees high performance at every level.

For more details or an in-surgery demonstration, contact Bien-Air UK Ltd on 01293550200 or email ba-uk@ bienair.com.

\section{FREE DVD FOR PAINLESS ANAESTHESIA}

General Medical have commissioned a Training DVD for Quicksleeper, the computer controlled local anaesthetic system that delivers profound anaesthesia quickly, easily and painlessly.

Available free of charge, the DVD describes the theory behind the technique and demonstrates the Quicksleeper in use for a variety of procedures. Having viewed it once or twice, you will be fully equipped to introduce the Quicksleeper into your practice and eliminate the painful ID block, palatal and infiltration injection procedures many adults and children fear.

Having first painlessly numbed the interdental papilla and underlying periosteum with just three or four drops of local, Quicksleeper enables dentists to perform osteocentral anaesthesia whereby a small amount of conventional local anaesthetic is placed in the pain-receptor-free spongy cancellous bone in between teeth. This delivers a profound anaesthesia almost instantaneously. Because it acts locally and does not create lingual anaesthesia or the "thick lip' associated with ID and mental blocks there is no risk of the patient biting the lip and it is safe to use bilaterally on the mandible.

It is possible to anaesthetise up to eight teeth with just one procedure, without the need for additional palatal or buccal injections. Completely painfree, it is effective even in the presence of pulpitis.

To request your free Training DVD call General Medical on 01380734990 or visit www. generalmedical.co.uk. 